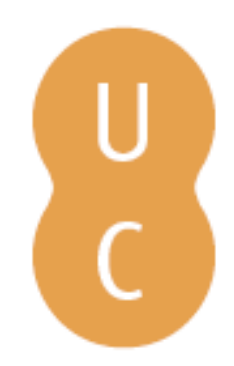

\title{
pombalina
}

\section{A dimensão do fator humano na segurança ferroviária: estados emocionais do profissional}

\author{
Author(s: $\quad$ Queirós, Cristina; Fonseca, Sérgio; Guimarães, Fabienne; Martins, Vítor \\ Published by: Imprensa da Universidade de Coimbra \\ Persistent URL: URI:http://hdl.handle.net/10316.2/41808 \\ DOI: $\quad$ DOI:https://doi.org/10.14195/978-989-26-1386-4_2 \\ Accessed : $\quad$ 18-Sep-2017 09:05:14
}

The browsing of UC Digitalis, UC Pombalina and UC Impactum and the consultation and download of titles contained in them presumes full and unreserved acceptance of the Terms and Conditions of Use, available at https://digitalis.uc.pt/en/terms_and_conditions.

As laid out in the Terms and Conditions of Use, the download of restricted-access titles requires a valid licence, and the document(s) should be accessed from the IP address of the licence-holding institution.

Downloads are for personal use only. The use of downloaded titles for any another purpose, such as commercial, requires authorization from the author or publisher of the work.

As all the works of UC Digitalis are protected by Copyright and Related Rights, and other applicable legislation, any copying, total or partial, of this document, where this is legally permitted, must contain or be accompanied by a notice to this effect. 
IMPRENSA DA UNIVERSIDADE DE COIMBRA

COIMBRA

UNIVERSITY

PRESS
LUCIANO LOURENÇO (COORD.)

ALCAFACHE

30 ANOS DEPOIS

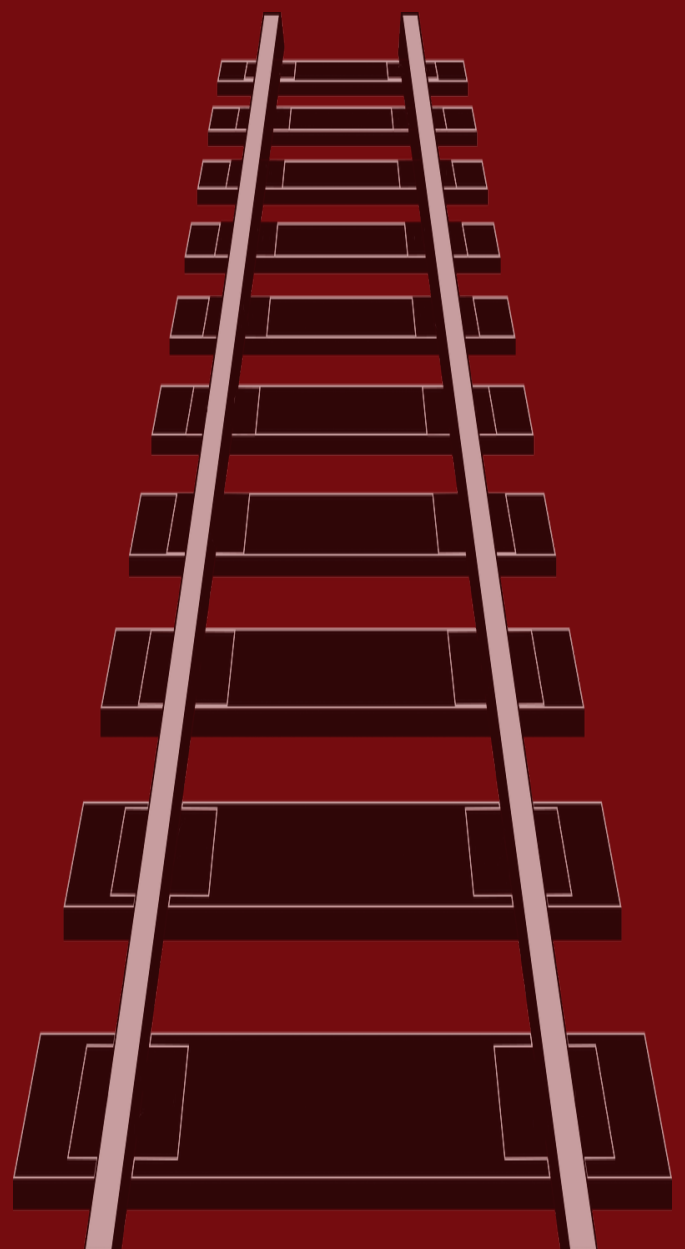




\title{
A DIMENSÁO DO FATOR HUMANO NA SEGURANÇA FERROVIÁRIA: ESTADOS EMOCIONAIS DO PROFISSIONAL HUMAN FACTOR DIMENSION IN RAILWAY SAFETY: PROFESSIONAL'S EMOTIONAL STATES
}

\begin{abstract}
Cristina Queirós
Faculdade de Psicologia e de Ciências da Educaçấo da Universidade do Porto cqueiros@fpce.up.pt

Sérgio Fonseca Faculdade de Psicologia e de Ciências da Educação da Universidade do Porto up201306558@fpce.up.pt

Fabienne Guimarães Faculdade de Psicologia e de Ciências da Educação da Universidade do Porto up201304095@fpce.up.pt

Vitor Martins

Sindicato Ferroviário da Revisão Comercial Itinerante sfrci@mail.telepac.pt
\end{abstract}

Sumário: A Agência Europeia para a Segurança e Saúde no Trabalho tem alertado para os riscos psicossociais no trabalho, entre os quais o stress crónico ou pós-traumáticos. Os trabalhadores da ferrovia estão expostos a inúmeras situaçóes geradoras de estados emocionais negativos no trabalho e trauma (ex: acidentes, colhidas, suicídios, agressóes de passageiros). Este estudo alerta para a importância dos estados emocionais no trabalho e para as consequências que podem ter na saúde do trabalhador. Pretende identificar as situaçóes stressantes a que os profissionais da ferrovia estão expostos e conhecer o impacto destas no stress pós-traumático e sintomas de stress. Os dados de 293 profissionais revelaram valores de stress e trauma de intensidade já 
moderada, presença de trauma em 32\% da amostra explicando este até $22 \%$ do stress, e acidentes com maior impacto traumático do que agressões. Sendo os trabalhadores da ferrovia forçados a interagir com passageiros num espaço fechado e a passar no mesmo local onde podem já ter tido acidentes, sugere-se mais atenção à gestão do stress e prevenção do trauma, de forma aos estados emocionais negativos não constituírem uma vulnerabilidade para a ocorrência de erros humano nos acidentes, afetando a segurança ferroviária.

Palavras-chave: Emoçóes, stress no trabalho, trauma, trabalhadores, ferrovia.

Abstract: European Agency for Safety and Health at Work is alerting to psychosocial risks at work, including chronic or post-traumatic stress. Railroad workers are exposed to many situations which can elicit negative emotional states at work and trauma (ex: accidents, train hits, suicides, assaults from passengers). This study alerts to the importance of emotional states at work and their consequences on the worker's health. The study aims at identifying the stressful situations to which railway workers are exposed and at knowing their impact on post-traumatic stress and stress symptoms. Data collected among 293 workers revealed moderate stress and trauma values, the presence of trauma in 32\% of the sample explaining trauma $22 \%$ of stress, and accidents having more traumatic impact than aggression. Railroad workers are forced to interact with passengers in an enclosed space and to circulate in the same place where they already had accidents. Thus, it should by payed more attention to stress management and trauma prevention, so that the negative emotional states do not become a vulnerability for human errors in accidents, affecting railroad safety.

Keywords: Emotions, job stress, trauma, workers, railway. 


\section{Introduçáa $^{1}$}

Nos últimos tempos, infelizmente, tem sido notícia a ocorrência de acidentes ferroviários graves, com vítimas mortais e feridos graves, como foi o caso do acidente de Setembro de 2016 com o descarrilamento do comboio Vigo-Porto em Porrińo, Espanha, a colisão entre comboios na estação de Alfarelos em Janeiro de 2013, a queda de uma automotora na Linha do Tua em Fevereiro de 2007, e o que foi, até agora, o mais trágico acidente ferroviários, a colisão do comboio Sud-Express em Moimenta-Alcafache, em Setembro de 1985. A nível internacional e próximo de nós, são recentes as recordaçôes dos descarrilamentos em Gent, Bélgica (Maio 2013), Bretigny-sur-Orge, França (Julho 2013), Santiago de Compostela, Espanha (Julho 2013) ou Eckwersheim, França (Novembro 2015), bem como das colisōes de Varsóvia, Polónia (Março 2012), Sloterdijk, Holanda (Abril 2012), Neuhausen, Suiça (Janeiro 2013), Pau-Bayenne, França (2014), Bad Aibling, Alemanha (Fevereiro 2016) ou Dudelange - Luxemburgo (Fevereiro 2017). Em Setembro de 2016 foram notícia acidentes na ferrovia no Reino Unido, Canadá, Argélia e New Jersey - Estados Unidos da América, e em Abril 2017 descarrilou perto de Coimbra um comboio de mercadorias, não se podendo esquecer os inúmeros acidentes relacionados com colhidas de pessoas ou veículos que diariamente ocorrem no mundo.

Perante a perda de vidas humanas, tratamento de feridos e prejuízos materiais, a preocupaçáo que surge é a das causas do acidente, sendo frequente a atribuição de erro humano. Por exemplo, na colisão de Bad Aibling, Alemanha (Fevereiro 2016) era noticiado ${ }^{2}$ que "um erro humano esteve na origem da colisão, esta terça-feira, entre dois comboios na Alemanha, que deixou dez mortos e 81 feridos, afirmaram vários media alemães", enquanto no acidente de Porriño, Espanha (Setembro 2016) surgia " Excesso de velocidade pode ter estado na origem do descarrilamento".

\footnotetext{
${ }^{1}$ Este texto corresponde à comunicação proferida pelos autores em 28 Maio 2016, no X Encontro Nacional de Riscos e II Jornadas Técnicas da Federaçáo dos Bombeiros do Distrito de Viseu.

2 http://www.jn.pt/PaginaInicial/Mundo/Interior.aspx?content_id=5022347\&page =-1 (9 Fevereiro 2016)

3 https://www.publico.pt/sociedade/noticia/excesso-de-velocidade-pode-ter-estado-na-origemdo-descarrilamento-1743694 (10 Setembro 2016)
} 
O fator humano nos acidentes de trabalho tem sido um tópico importante e, recentemente, Kumar e colaboradores (2016) alertaram que apesar de todos os desenvolvimentos da tecnologia no sentido de mecanizar tarefas e minimizar o erro humano, este ainda ocorre e deve ser discutido e prevenido para uma melhoria constante da segurança no trabalho. Os autores enumeram como principais categorias do erro humano a sonolência e desatenção por rotina, os enganos ou lapsos por falhas de memória, os erros pela necessidade de tomada de decisão imprevista ou falha de conhecimentos, e por fim, os incumprimentos ou violaçóes das normas em vigor por necessidade de reagir a um imprevisto, por excesso de confiança, distração ou características individuais. Também Moura e colaboradores (2015) reforçam a necessidade de compreender como o fator humano determina os acidentes no trabalho, nomeadamente na atual e complexa interação homem-máquina, devendo a tecnologia ser desenhada para minimizar o erro humano. No que se refere aos acidentes na ferrovia, Baysari e colaboradores (2009) classificaram os erros humanos neste tipo de acidentes como relacionados com a tarefa ou com o desempenho, referindo por exemplo: erros de manobra na paragem do comboio; excesso de velocidade; problemas com o material, infraestrutura, documentos; problemas com outros envolvidos (ex.: veículo ou pessoa em situação imprevista na linha); situações imprevistas não contempladas na rotina das tarefas; cansaço e falta de atenção em tarefas rotineiras; problemas de comunicação com central ou outros funcionários implicados na tarefa; problemas de aplicação dos conhecimentos na tomada de decisão; falta de informaçáo ou problemas nos procedimentos; problemas de perceção dos detalhes da situação; e por fim problemas pessoais ou problemas de interação com colegas.

Em qualquer das situaçóes descritas, a dimensão individual do trabalhador tem um papel importante, pois é este que toma uma decisão que pode ter como consequência o acidente, estimando-se que $90 \%$ dos acidentes são devido a erro humano (Yeow et al., 2014). Apesar dos avanços na segurança no trabalho e da preocupação com os conhecimentos do trabalhador para a realização da tarefa, pouca valorização tem sido dada à influência do stress, fadiga e rotina nos erros humanos que terminam em acidentes (Yeow et al., 2014). Contudo, 
o stress tem tido um interesse crescente como um dos riscos psicossociais emergentes no contexto laboral (EU-OSHA, 2006), nomeadamente na interação homem-máquina como é o caso do transporte ferroviário. De facto, em 2005 a Agência Europeia para a Segurança e Saúde no Trabalho (EU-OSHA, 2005, p.5) referia a necessidade de "uma abordagem integrada mais holistica, promovendo uma cultura de prevenção que se refletiu na nova iniciativa Locais de Trabalho Saudáveis»", alertando em 2006 que "os ambientes de trabalho estão em constante mudança sob a influência de novas tecnologias e de condiçóes económicas, sociais e demográficas que se vão alterando", sendo necessário "antecipar os novos riscos emergentes" (EU-OSHA, 2006, p.1). Pouco depois, incluiu o stress como um risco emergente (EU-OSHA, 2010, 2013a) nas suas prioridades para a segurança e saúde ocupacional 2013-2020. Em 2014 lançou a campanha "Locais de trabalho saudáveis" (EU-OSHA, 2014a), reformulada em 2016 para “2016/17: Locais de trabalhos saudáveis para todas as idades" (EU-OSHA, 2016a), que pressupóe que o trabalhador se mantenha no seu local de trabalho até á idade de reforma, e esteja em boas condiçóes físicas e psicológicas. Recorde-se que já foram efetuados levantamentos para estimar o custo dos acidentes e problemas de saúde relacionados com o trabalho, bem como com os custos do stress e dos riscos psicossociais relacionados com o trabalho (EU-OSHA, 2013b, 2014b), enumerando custos com a produtividade, com perdas de qualidade de vida, e custos administrativos e de seguro, que afetam não só o trabalhador e sua família, mas também a empresa e sobretudo a sociedade, quando, como é o caso da ferrovia, há danos para os passageiros. Estima-se que os custos dos acidentes sejam na ordem de 1 a 4,8\% do PIB do país (EU-OSHA, 2013b, p.6) e que os riscos psicossociais afetem o bem estar mental de $28 \%$, ou seja, cerca de 5,6 milhões de trabalhadores europeus (EU-OSHA, 2014b, p.4).

Sendo o stress considerado um risco psicossocial e afetando $14 \%$ dos trabalhadores europeus (EU-OSHA, 2014b, p.4), convém lembrar que em termos laborais constitui um desafio para a saúde ocupacional e para a segurança no trabalho, pois em 2013 cerca de metade dos trabalhadores europeus considerava o stress uma situação comum no local de trabalho, contribuindo este para cerca de $50 \%$ dos dias de trabalho perdidos (EU-OSHA, 2013a, 2014a). Contudo o 
stress é considerado estigmatizante enquanto problema de saúde mental sendo associado a uma falha individual do trabalhador, apesar de poder ser controlado se abordado numa perspetiva organizacional, ou seja, como outro qualquer risco de saúde e segurança no local de trabalho que resulte de deficiências na conceção, organização e gestão do trabalho ou de um contexto social de trabalho problemático cuja consequência se traduza em efeitos negativos a nível psicológico, físico e social, como por exemplo o stress no trabalho, esgotamento, depressão, doenças músculo-esqueléticas ou gastrointestinais e conflitos laborais, absentismo ou presentismo (EU-OSHA, 2013a).

No que se se refere aos profissionais da ferrovia, estes estão expostos a múltiplos fatores de stress no trabalho, bem como a situaçóes de acidentes ou incidentes críticos, cujo impacto psicológico pode ser de tal forma elevado que configura um quadro de stress pós-traumático (Cocks, 1989; Doroga \& Baban, 2013, Mehnert et al., 2012). Destacam-se situaçóes como condições físicas de trabalho (ex. ergonomia dos equipamentos, falta de local de descanso entre turnos), horários e gestão dos turnos, insegurança na interação com passageiros e ameaças ou agressóes por parte destes, bem como colhidas de pessoas e veículos (com especial destaque para os suicídios na linha), estas últimas desencandeadoras de estados emocionais negativos crónicos (Queirós, Fonseca, Guimarães \& Martins, 2016).

Sabe-se que, no que se refere especificamente ao stress pós-traumático, o tipo de trabalho pode influenciar a incidência deste tipo de stress, pois alguns profissionais (ex: militares, polícias, bombeiros, motoristas, profissionais de saúde ou de socorro) estão expostos a graves traumas psicológicos, agravados quando ocorrem situaçóes sucessivas ou ao longo do acumular da experiência profissional (Saberi et al., 2013). No que se refere aos profissionais do sector do transporte, verificou-se que motoristas comerciais que estiveram envolvidos em acidentes ou assistiram a acidentes de outros motoristas, têm maior risco de desenvolver este tipo de stress, o que pode ter como consequência mais erros humanos e mais acidentes de trabalho (Saberi et al., 2013). Note-se que, contrariamente aos elementos de equipas de emergência médica e salvamento, no setor ferroviário os profissionais não são selecionados para a sua função tendo em consideração 
a resiliência. Contudo, imediatamente após um acidente ou suicídio na linha, o maquinista tem a responsabilidade de gestáo dos passageiros no rescaldo da situaçáo, sendo, como tal, difícil náo ser afetado psicologicamente (Lunt \& Hartley, 2004). Pode ainda culpar-se pela incapacidade de agir (ex. conseguir parar o comboio a tempo) e ser confrontado com as imagens impressionantes das pessoas na via ou corpos mutilados, bem como odores intrusivos durante e após o acontecimento traumático (Mehnert et al., 2012). Em Portugal, entre 2004 e 2014, foram contabilizadas (IMT, 2015) 1077 pessoas envolvidas (vítimas) em acidentes ferroviários e ainda 473 suicídios (média anual de 47 mortes).

Para além dos acidentes, os profissionais da ferrovia são alvo de agressão no local de trabalho por parte de clientes, especialmente os que têm contacto direto com o cliente, sendo esta agressáo definida como um comportamento destinado a prejudicar ou perturbar o prestador de serviço (Dormann \& Zapf, 2004; Dursun \& Aytac, 2014) que pode ocorrer em áreas variadas como comércio, restauração, hóteis, companhias áereas e ferroviárias, call centers ou setor bancário (Bedi \& Schat, 2007). Em 2002, Boyd estimava que 53\% dos trabalhadores nas empresas de transporte aéreo e ferroviário já estiveram expostos ao insulto verbal nos últimos 12 meses, podendo a agressão psicológica (gritos, gestos, insultos, ameaças, hostilidade, etc.) ou a violência física afetar os trabalhadores tanto a nível físico como psicológico (Richards \& Schat, 2007). Estas situaçóes levam a que o trabalhador se sinta desvalorizado e os níveis de depressão e stress aumentam (Boyd, 2002; Bedi \& Schat, 2007; Grandey, Dickter \& Sin, 2004), bem como diminui a satisfação e a concentração na tarefa, aumenta a desmotivação, o absentismo, a rotatividade das funçôes e a ausência por motivo de doença (Dursun \& Aytac, 2014; Yagil, 2008).

Pelo exposto, constata-se que o trabalhador da ferrovia tem uma atividade profissional stressante, na qual um erro humano pode ter trágicas consequências e onde por vezes o controle para evitar um acidente é nulo, como por exemplo, perante um suicídio na linha. Estão pois criadas as condições para o aparecimento do stress pós-traumático (Vaz Serra, 2003), também designado como perturbação pós-stress traumático ou, internacionalmente, como PTSD ou Post-traumatic Stress Disorder (Pereira \& Monteiro-Ferreira, 2003). Note-se 
que para uma situação provocar stress pós-traumático a pessoa tem de se confrontar com uma experiência "além do limite das experiências com que a vida confronta qualquer cidadão comum" (Pereira \& Monteiro-Ferreira, 2003, p.15), sendo esta desencadeadora de um forte impacto emocional, capaz de levar a uma fixação no acontecimento, e como tal, a uma recordação que não diminui com o tempo e perturba a vivência diária. Esta situação traumática pode ser comparada a uma ferida emocional que provoca desequilíbrio na vítima e a deixa em sofrimento, prejudica a sua capacidade de adaptação e de enfrentar a situação devido à intensidade e imprevisibilidade com que ocorre, e gera desorganização psicológica que impede a compreensão adequada e a atribuição de significado correta à situação ocorrida (Pereira \& MonteiroFerreira, 2003).

De acordo como o Manual de Diagnóstico e Estatístico de Transtornos Mentais DSM-V (APA, 2014), os transtornos relacionados com trauma apresentam como critério de diagnóstico a exposição da vítima a um evento traumático ou stressante (ex. ameaça de morte ou lesão grave), vivenciado diretamente ou testemunhado pessoalmente noutras pessoas, bem como o facto de a vítima poder tomar conhecimento de um incidente crítico que ocorreu com um familiar ou amigo próximo de forma violenta ou acidental, ou ter exposição repetida ou extrema a detalhes aversivos do evento traumático (ex. socorristas que recolhem partes de corpos humanos, polícias repetidamente expostos a situaçóes de violência, e acrescentamos nós, profissionais da ferrovia expostos a colhidas ou suicídios na linha, em que qualquer uma destas situaçóes provoca na vítima de trauma um stress elevado e um disfuncionamento na interação social ou na sua capacidade de trabalho).

É importante ter ainda em consideração os sintomas comportamentais que acompanham o stress pós-traumático (APA, 2014), os quais devem durar mais de um mês após o acontecimento (embora possam também surgir cerca de 6 meses depois), considerando-se que até um mês são uma reação normal ao ocorrido. Estes sintomas são agrupados em quatro categorias utilizadas para definir a ocorrência de stress pós-traumático (Pereira \& Monteiro-Ferreira, 2003): pensamentos intrusivos, evitamento, pensamentos e humor 
negativos, e hiperativação. Os pensamentos intrusivos consistem no reviver o acontecimento de forma inesperada e involuntária, através de memórias frequentes, pesadelos ou momentos em que a vítima pensa estar de novo no acontecimento. São acompanhados de ansiedade, sofrimento psicológico e reaçóes físicas intensas quando surge algo semelhante ao acontecimento traumático, e vítima pode ter alterações na perceção da realidade e forma de estar com os outros. O evitamento consiste no esforço para evitar recordaçóes, pensamentos ou sentimentos sobre o acontecimento, podendo existir a recusa de contacto com pessoas, locais, conversas, atividades, objetos ou situaçóes que possam provocar recordaçóes do acontecimento. No limite, a vítima pode sentir-se anestesiada afetivamente e incapaz de sentir emoçóes. Os pensamentos e humor negativos referem-se ao facto de, após o acontecimento, a vítima se sentir incapaz de recordar aspetos importantes da situação, tendo expectativas negativas persistentes e exageradas a respeito de si mesmo, dos outros e do mundo. Por vezes culpa-se pelo que aconteceu e sente mais emoçốes negativas do que positivas, tendo tendência a isolar-se de todos. A hiperativação consiste em reaçóes fisiológicas intensas que geram momentos de irritação, raiva ou agressividade contra pessoas/objetos, comportamentos imprudentes ou autodestrutivos, hipervigilância ou resposta de sobressalto exagerada, problemas de concentração e perturbaçôes do sono (sono agitado ou dificuldade em iniciar ou manter o sono). A vítima é incapaz de diminuir o seu grau de alerta, o qual está sempre no nível máximo pois a pessoa vê ameaças em tudo e todos.

A percentagem de pessoas afetadas por stress pós-traumático é muito variada e depende do tipo de situaçáo e de muitos outros fatores, como o contexto de socorro, o apoio após o acontecimento, as condiçôes físicas, etc. (Vaz Serra, 2003). Contudo Maia e Fernandes (2003, pp.41-43) citam vários estudos publicados desde a década de noventa e referem em sobreviventes de: terramoto no México 30\%; terramoto na Arménia 74\%; inundação por barragem 59\%; acidentes rodoviários graves 12\%; vítimas de violação $57 \%$ a $94 \%$; veteranos de guerra 25 a 30\%; vítimas de ataques terroristas $20 \%$ a $40 \%$; bombeiros expostos a desastres naturais $16 \%$. 
Mais recentemente, outros autores (Adams et al., 2014; Green et al., 2015; Johannesson et al., 2006; Kilpatrick et al., 2013; North et al., 2015; Ranney et al., 2016; Sachser \& Goldbeck, 2016; Wilson, 2015) referem: 53\% em familiares de vítimas de desastre aéreo; $51 \%$ em sobreviventes de desastres; $48 \%$ em sobreviventes de acidentes ou incêndios; $32 \%$ a $52 \%$ em familiares de vítimas de acidente/desastre/ violência; $53 \%$ em vítimas e $33 \%$ em testemunhas de crime sexual ou com violência física; 68 a 73\% em combatentes no Iraque ou Afeganistão; $31 \%$ em sobreviventes dos atentados de 11 de Setembro EUA; $13 \%$ a $22 \%$ em profissionais de socorro; $7 \%$ em adolescentes sobreviventes de um tornado nos EUA; $61 \%$ a $81 \%$ em adolescentes vítimas de violência sexual ou física ou perdas traumáticas; $23 \%$ em adolescentes expostos a violência na comunidade, violência entre pares ou cyberbullying. Estas percentagens são valores muito globais que podem variar ainda conforme o momento da recolha de dados seja próximo do acontecimento ou decorra meses ou anos depois. Mais recentemente, Brancu e colaboradores (2016) referiram percentagens globais entre $13 \%$ e $16 \%$, conforme o rigor dos estudos e os critérios de medição.

Nos profissionais da ferrovia existem poucos estudos e a percentagem de stress pós-traumático tem sido referida como 6\% na Roménia (Doroga \& Baban, 2013), 8\% em Portugal (Lemos, 2013) e 14\% na Alemanha (Mehnert et al., 2012). Este trabalho, partiu de um estudo conjunto realizado entre 2015 e 2016 pela Faculdade de Psicologia e de Ciências da Educação da Universidade do Porto e pelo Sindicato Ferroviário da Revisão Comercial Itinerante, no qual foram analisados a satisfaçáo e motivação para o trabalho, suporte familiar e de colegas, conflito entre trabalho e família, absentismo, stress, burnout e trauma (Queirós et al., 2016), e alerta, na ferrovia, para a importância dos estados emocionais no trabalho e para as consequências que podem ter na saúde do trabalhador. Tem como objetivo identificar as situaçóes stressantes a que os profissionais da ferrovia estáo expostos e conhecer o impacto destas a nível do stress pós-traumático, sintomas corporais de stress e estados emocionais. 


\section{Método}

\section{Participantes}

Foram inquiridos 293 profissionais da ferrovia, sendo $95 \%$ do sexo masculino, $66 \%$ com $12^{\circ}$ ano, $79 \%$ casados, $85 \%$ com filhos, $60 \%$ com experiência de acidentes na ferrovia. Eram provenientes $64 \%$ da zona norte (36\% do resto do país), e $80 \%$ tinham funções de operador de revisão e venda (circulam nos comboios) e $20 \%$ funçóes de operador de venda e controle (estâo nos postos fixos de cada estaçáo). A idade variou entre 27 e 62 anos $(M=43,6$ anos e $\mathrm{DP}=6,136)$, enquanto a experiência profissional variou entre 1 e 36 anos $(\mathrm{M}=17,7$ anos e $\mathrm{DP}=6,897)$.

\section{Instrumentos}

No âmbito do projeto mais vasto já referido (Queirós et al., 2016) foi preparado um questionário com questôes sociodemográficas e profissionais, bem como avaliado o impacto traumático de situações stressantes através da Impact of Event Scale Revised (IES-R, Horowitz et al.1979; Cauby et al., 2012) e o stress, através do Cohen-Hoberman Inventory of Physical Symptoms (CHIPS, Cohen \& Hoberman, 1983; Queirós et al., 2016). O impacto de incidentes stressantes foi avaliado por algumas questôes abertas que tentaram perceber em quantos acidentes ou outras situações no trabalho os inquiridos estiveram envolvidos, e de que forma se sentiram afetados. A IES-R é composta por 22 itens avaliados numa escala de Likert de 5 pontos (0 para nunca e 4 para muitas vezes), organizados nas dimensóes: pensamentos intrusivos (memórias do acontecimento stressante), evitamento (recusar pensar ou falar do acontecimento) e hperativação (estado de alerta fisiológico e psicológico constante), sendo possível ainda calcular um resultado total de stress pós-traumático. $\mathrm{O}$ CHIPS é composto por 33 sintomas avaliados numa escala de Likert de 5 pontos (0 para não incomodou nada e 4 para incomodou muito), podendo ainda ser 
contabilizados quantos sintomas incomodaram (sintomas avaliados com valor superior a zero, variando de 0 a 33 sintomas) e o total de incómodo (variando de 0 a 132, ou seja, 33 sintomas com 4 como grau máximo de incómodo).

\section{Procedimentos}

A recolha de dados foi iniciada após autorização formal e divulgação do estudo pelo Sindicato Ferroviário da Revisão Comercial Itinerante, com os questionários impressos distribuídos por esta entidade, sendo preenchidos de forma anónima e confidencial entre Março e Julho de 2015 e, posteriormente, devolvidos em envelope à FPCEUP. Foi apresentado o consentimento informado e cumpridas as regras éticas e deontológicas para este tipo de estudo empírico. Em seguida os dados foram introduzidos no programa IBM SPSS Statistics versão 21 e a análise estatística foi efetuada de acordo com os pressupostos recomendados por vários autores (Bryman \& Cramer, 2003; Field, 2009; Maroco, 2010) para este tipo de amostra, variáveis e objetivos do estudo, correspondendo a uma análise descritiva (frequência, percentagem, média e desvio-padrão), comparativa ( $t$ de student para grupos independentes), correlacional ( $\mathrm{R}$ de Pearson) e de regressão (método Enter).

\section{Resultados}

Os resultados obtidos revelam, quanto ao impacto de incidentes stressantes (QuADro I), que este varia, nas suas diferentes dimensóes, entre 1 e 1,19 numa escala de 0 a 4, sendo considerado moderado, enquanto o total de trauma é moderado com média de 24,29 para um máximo de 88 pontos. Contudo, usando o ponto de corte definido por Weiss (2007) como 33 para indicador de stress pós-traumático, foi encontrada uma percentagem de $31,9 \%$ com valores entre 33 e 88, incluindo 5 casos acima de 80. Este valor é bastante superior aos 6 a 14\% referidos por Doroga e Baban (2013), Lemos (2013) e Mehnert 
e colaboradores (2012). Quanto aos sintomas físicos de stress verifica-se que a sua média é de 1,31 numa escala de 0 a 4 de incómodo, ou seja, tem um nível moderado-baixo, com presença de cerca de 19 sintomas em 33 e com um grau de incómodo baixo (média de 42 para um máximo possível de 132). Assim, em síntese pode-se dizer que estes profissionais apresentam já alguns sintomas físicos e psicológicos imediatos de stress, e o impacto de trauma é já moderado, sugerindo um processo de mal-estar com sintomas típicos de desgaste profissional ao longo do tempo e náo de situaçôes imediatas (expressas nos sintomas de stress), parecendo os profissionais ir buscar forças e motivação mais fora do local de trabalho do que dentro deste (dados recolhidos nas questóes abertas sobre situaçóes stressantes no trabalho).

QUADRO I - Médias por dimensão avaliada no stress e trauma na amostra total.

TABLE I - Averages by dimension measured in stress and trauma on the overall sample.

\begin{tabular}{|l|l|l|l|l|}
\hline \multicolumn{1}{|c|}{$\begin{array}{c}\text { Dimensão por questionário } \\
\text { (escala de avaliaçáo) }\end{array}$} & Mínimo & \multicolumn{1}{|c|}{ Máximo } & \multicolumn{1}{|c|}{ Média } & \multicolumn{1}{|c|}{$\begin{array}{c}\text { Desvio } \\
\text { Padrão }\end{array}$} \\
\hline Pensamentos Intrusivos (0 a 4) &, 00 & 4,00 & 1,19 & 0,96 \\
\hline Evitamento &, 00 & 4,00 & 1,15 & 0,91 \\
\hline Hiperativação &, 00 & 4,00 & 1,01 & 0,96 \\
\hline Total Trauma (0 a 88) &, 00 & 88,00 & 24,29 & 19,57 \\
\hline Incómodo Sintomas Stress (0 a 4) &, 00 & 3,67 & 1,31 & 0,81 \\
\hline Total sintomas (0 a 33) &, 00 & 33,00 & 19,21 & 9,33 \\
\hline Total incómodo (0 a 132) &, 00 & 121,00 & 42,31 & 26,30 \\
\hline
\end{tabular}

As situaçóes enumeradas como mais stressantes e potenciadoras de trauma foram os acidentes (incluindo aqui colisōes, colhidas e suicídios) e as agressōes e ameaças no local de trabalho por parte de passageiros, respetivamente com $59 \%$ e $15 \%$, existindo $26 \%$ dos participantes que refere situaçôes variadas como conflitos com colegas ou superiores, falta de meios para agir em caso de acidente, falta de comunicação por atrasos o que motiva revolta e ameaças dos passageiros, ou problemas entre passageiros dentro do comboio (ex: quedas, doenças, conflitos, roubos, agressóes). Note-se que as situaçóes descritas ocorreram entre 1980 e 2015, assistindo-se a um aumento de relatos já depois de 
2000, sendo notório o aumento a partir de 2011, com especial ênfase em 2014 (Queirós et al., 2016). Comparando apenas o impacto stressante de acidentes e agressōes (QUADro II), verifica-se que os sintomas de stress não apresentam diferenças significativas, enquanto no stress pós-traumático apenas a hiperactivação não diferencia os dois tipos de situação, apresentando os acidentes valores significativamente superiores, comprovando-se assim o impacto traumático dos acidentes, conforme já descrito nos estudos de Cocks (1989), Mehnert e colaboradores (2012), Doroga e Baban (2013), Saberi e colaboradores (2013) e Lunt e Hartley (2004).

QUADRO II - Comparação de médias do stress e trauma em função da situação.

TABLE II - Average comparison of stress and trauma according to the situation.

\begin{tabular}{|c|c|c|c|c|}
\hline $\begin{array}{c}\text { Dimensáo por questionário } \\
\text { (escala de avaliaçáo) }\end{array}$ & $\begin{array}{l}\text { Acidente } \\
\mathrm{N}=172\end{array}$ & $\begin{array}{l}\text { Agressáo } \\
\mathrm{N}=44\end{array}$ & $\begin{array}{c}\mathbf{t} \\
\text { Student }\end{array}$ & $\mathbf{p}$ \\
\hline Pensamentos Intrusivos ( 0 a 4$)$ & 1,39 & 0,92 & 2,652 &, $009^{* *}$ \\
\hline Evitamento & 1,31 & 0,88 & 2,643 &, $009^{* *}$ \\
\hline Hiperativação & 1,15 & 0,84 & 1,738 & ,084 \\
\hline Total Trauma (0 a 88$)$ & 28,22 & 18,71 & 2,640 &, $009^{* *}$ \\
\hline Incómodo Sintomas Stress (0 a 4) & 1,36 & 1,25 &, 806 & 421 \\
\hline Total sintomas (0 a 33) & 19,95 & 18,91 & ,683 & 495 \\
\hline Total incómodo (0 a 132) & 43,63 & 41,32 &, 546 &, 585 \\
\hline
\end{tabular}

A análise correlacional entre idade, anos de serviços, trauma e stress (QUADro III) revelou que a idade não apresenta correlaçóes significativas com o trauma nem com o stress, enquanto os anos de serviço revelam que quanto mais são, maior o incómodo de stress e mais os pensamentos intrusivos, hiperativaçấo e total de trauma. Foram também encontradas correlaçóes positivas entre todas as dimensôes do trauma e do stress, revelando que quanto maior um, maior o outro.

Por fim, a análise de regressão relativa ao poder explicativo do trauma e variáveis sociodemográficas e profissionais nos níveis de stress atuais (QUADRo IV), revelou que as características individuais não têm valor preditivo, mas o trauma explica $22 \%$ do incómodo dos sintomas de stress, $15 \%$ do total de sintomas e $21 \%$ do total de incómodo. 
QUADRO III - Correlação entre idade, anos de serviços, trauma e stress.

TABLE III - Correlation between age, professional experience years, trauma and stress.

\begin{tabular}{|l|l|l|l|l|l|l|}
\hline \multicolumn{1}{|c|}{ Dimensáo } & Idade & $\begin{array}{c}\text { Anos } \\
\text { serviço }\end{array}$ & $\begin{array}{c}\text { Pens. } \\
\text { Intrusivos }\end{array}$ & Evitamento & Hiperativaçáo & $\begin{array}{c}\text { Total } \\
\text { Trauma }\end{array}$ \\
\hline Pens. Intrusivos &, 036 &, $151^{*}$ & & & & \\
\hline Evitamento &,- 046 &, 092 & & & & \\
\hline Hiperativaçáo &, 048 &, $137^{*}$ & & & & \\
\hline Total Trauma &, 011 &, $136^{*}$ & & & &, $434^{* *}$ \\
\hline Incómodo S. Stress &, 083 &, $136^{*}$ &, $428^{* *}$ &, $341^{* *}$ &, $359^{* *}$ &, $353^{* *}$ \\
\hline Total sintomas &,- 008 &, 096 &, $354^{* *}$ &, $285^{* *}$ &, $416^{* *}$ &, $400^{* *}$ \\
\hline Total incómodo &, 051 &, 105 &, $407^{* *}$ &, $313^{* *}$ &, 40 & \\
\hline
\end{tabular}

${ }^{*} \mathrm{p} \leq 0,050 \quad{ }^{* *} \mathrm{p} \leq 0,010$

QUADRO IV - Regressão (Enter) do trauma e características sociodemográficas nos sintomas de stress.

TABLEIV - Regression (Enter) of trauma and sociodemographic caractheristics on stress symptoms.

\begin{tabular}{|l|l|l|l|l|}
\hline \multirow{2}{*}{ Variável Dependente } & \multicolumn{1}{|c|}{$\begin{array}{c}\text { Variáveis } \\
\text { Preditoras }\end{array}$} & \multicolumn{1}{|c|}{$\mathbf{R}^{2}$} & $\mathbf{R}^{2}$ change & \multicolumn{1}{c|}{$\mathbf{F}($ sig) } \\
\hline \multirow{2}{*}{$\begin{array}{l}\text { Incómodo Sintomas } \\
\text { Stress }\end{array}$} & Trauma &, 215 &, 215 & $16,032(, 000)^{* * *}$ \\
\cline { 2 - 6 } & Sociodemográficas &, 263 &, 048 & $1,311(, 219)$ \\
\hline \multirow{2}{*}{ Total sintomas } & Trauma &, 147 &, 147 & $10,087(, 000)^{* * *}$ \\
\cline { 2 - 6 } & Sociodemográficas &, 210 &, 063 & $1,615(, 096)$ \\
\hline \multirow{2}{*}{ Total incómodo } & Trauma &, 204 &, 204 & $15,012(, 000)^{* * *}$ \\
\cline { 2 - 6 } & Sociodemográficas &, 258 &, 058 & $1,479(, 141)$ \\
\hline \multirow{2}{*}{$\mathrm{p} \leq 0,050 *{ }^{* *} \mathrm{p} \leq 0,010$} & ${ }^{* * *} \mathrm{p} \leq 0,001$ & & \\
\hline
\end{tabular}

\section{Conclusão}

Os resultados obtidos neste estudo, apesar de indicarem baixo/moderado incómodo e presença de sintomas de stress, e moderado nível de stress pós-traumático, apresentam como resultados principais o facto de o trauma e incómodo dos sintomas de stress aumentarem em função dos anos de serviço, sugerindo um acumular de situaçóes stressantes, bem como o facto de existirem 
$32 \%$ dos participantes com presença de trauma (valores superiores a outros estudos na Europa), e o facto de o trauma estar positivamente correlacionado com sintomas de stress e explicar $15 \%$ a $22 \%$ destes sintomas. Se atendermos a que o stress pode ter como consequência o erro humano e que este está na base de 90\% dos acidentes (Yeow et al., 2014), entâo a presença de stress pós-traumático nestes profissionais pode constituir um risco acrescido de acidentes ao desencadear a vulnerabilidade ao stress. Um outro resultado importante é o facto de as situaçóes stressantes se poderem agrupar sobretudo nas categorias relativas a "acidentes" ou a "agressóes ou ameaças" no local de trabalho.

Ora, ao contrário de outros profissionais do sector dos transportes, os trabalhadores da ferrovia são forçados a interagir com passageiros num espaço fechado e a passar no mesmo local onde podem ter tido acidentes, colhidas ou suicídios. Acresce que, comparados com outros profissionais nos quais o stress pós-traumático pode ocorrer em contextos de trabalho, o trabalhador da ferrovia dificilmente deixa de poder evitar esse local devido à logística e burocracia organizacional das empresas ferroviárias que tornam o processo de mudança de local de trabalho, raramente solicitado e extremamente moroso. Perpetuando-se ao longo de vários anos, na maioria dos casos, o trabalhador é obrigado a confrontar-se com o mesmo local de acidente ou suicídio, constantemente revivendo a situação, o que pode potenciar os sintomas.

É então fundamental que se valorize que os estados emocionais negativos no trabalho constituem um processo de esgotamento ao longo do tempo por falta de recursos psicológicos, que culmina num estado (designado como burnout) e que implica enorme sofrimento psicológico para o trabalhador e problemas na sua produtividade e qualidade dos serviços prestados (Maslach \& Leiter, 2016), incluindo aumento do erro humano nos acidentes. Para tal, são necessários estudos e intervençôes sobre a saúde ocupacional dos profissionais da ferrovia, à semelhança de outros países (Cothereau et al., 2004; Cox et al., 2003; Doroga \& Baban, 2013; Sage et al., 2016; Vatshelle \& Moen, 1997), bem como a criação de programas de prevençáo e tratamento direcionados para as necessidades dos profissionais do sector ferroviário, com características distintas de outros trabalhadores. Esta ideia já foi operacionalizada na década de 90 pela British Rail (Williams et al., 1994) e, recentemente Bardon e Mishara (2015) sugeriram que se constitua um protocolo 
de atuação composto por açôes de prevenção pré-incidente (ex: acidentes, suicídios na linha ou agressóes de passageiros), açóes de intervençáo no momento do incidente e resposta a curto prazo após o incidente. Seriam notórios os benefícios no trabalhador, na segurança dos passageiros e na produtividade da empresa, constituindo ainda um modelo de boas práticas. Contudo, implicaria integrar estruturas e práticas organizacionais já existentes, bem como formar e dotar os profissionais (que não são especialistas de emergência mas têm de agir nos incidentes críticos), de competências individuais que lhes permitisse agir melhor junto dos passageiros e prevenir neles próprios ou nos colegas o impacto negativo destes incidentes críticos e gerir os seus sintomas de stress. Conforme a Agência Europeia para a Segurança e Saúde no Trabalho (EU-OSHA, 2005, 2006, 2014a, 2016b) tem vindo a alertar, "[...] com a abordagem correta, os riscos psicossociais e o stress no trabalho podem ser prevenidos e geridos com sucesso, independentemente da dimensão ou tipo de empresa, representando uma obrigação moral e um bom investimento para as organizaçôes, sendo incentivado no Pacto Europeu para a Saúde Mental e Bem-Estar, a implementação de medidas voluntárias suplementares para a promoção do bem-estar mental e da saúde ocupacional, nas quais os trabalhadores estejam envolvidos, pois têm uma melhor perceşão dos problemas que podem ocorrer no local de trabalho".

Contudo, mesmo com a melhoria da segurança dos transportes, os acidentes são imprevisíveis e continuam a acontecer, mantendo-se na sua génese o erro humano ${ }^{4}$, a falha do material circulante e a deficiência na infraestrutura ferroviária (ex. sinalização, via, etc.), sem esquecer a hipótese externa de uma ação terrorista (ex. Atocha, Espanha em 11 de Março de 2004; Sao-Petersburgo, Rússia, em Abril 2017). A elevada taxa de ocupação de passageiros e a maior frequência de transportes ferroviários (ex. comboio e metropolitano) leva a que os profissionais da ferrovia tenham uma responsabilidade acrescida, o que implica que o seu bem-estar psicológico deve estar acautelado em termos de pré-incidente e de pós-incidente, tentando prevenir o trauma e seu impacto ao lingo do tempo.

Para terminar, não podemos deixar de alertar que apesar deste estudo se ter centrado nos profissionais da ferrovia, os acidentes com comboios implicam a mobi-

\footnotetext{
${ }^{4}$ https://www.publico.pt/sociedade/noticia/excesso-de-velocidade-pode-ter-estado-na-origemdo-descarrilamento-1743694 (10 Setembro 2016).
} 
lização de inúmeros profissionais de socorro, também estes expostos a situaçôes potencialmente traumáticas e com elevada probabilidade de vivenciarem exatamente os sintomas de trauma já descritos e de sentirem o seu impacto muitos anos depois. Foi este o caso com os testemunhos emocionantes, e, sobretudo, emocionados, de vários profissionais envolvidos no acidente do comboio Sud-Express em Moimenta-Alcafache, em Setembro de 1985, apresentados no X Encontro Nacional de Riscos e II Jornadas Técnicas da Federação dos Bombeiros do Distrito de Viseu. As suas emoçôes ao relatarem a sua participação sugerem que as memórias dessa situação traumática ainda estâo bem vivas, 31 anos depois deste trágico acidente, demonstrando que, tal como Rabjohn (2012) afirma, existem custos humanos quando se presta socorro, e estes devem passar a ser considerados no futuro.

\section{Bibliografia}

Adams, Z.W., Sumner, J.A., Danielson, C.K., McCauley, J.L., Resnick, H.S., Grös, K., Paul, L., Welsh, K. \& Ruggiero, K. J. (2014). Prevalence and predictors of PTSD and depression among adolescent victims of the spring 2011 tornado outbreak. Journal of Child Psychology and Psychiatry, 55(9), 1047-1055.

APA, American Psychiatric Association (2014). Manual de Diagnóstico e Estatístico de Transtornos Mentais DSM-5. Porto Alegre: Artmed.

Bardon, C. \& Mishara, B. (2015). Development of a Comprehensive Programme to Prevent and Reduce the Negative Impact of Railway Fatalities, Injuries and Close Calls on Railway Employees. Journal of Occupational Rehabilitation, 25, 557-568.

Baysari, M., Caponecchia, C., McIntosh, A. \& Wilson, J. (2009). Classification of errors contributing to rail incidents and accidents: A comparison of two human error identification techniques. Safety Science, 47, 948-957.

Bedi, A. \& Schat, A. C. H. (2007). Customer Aggression: A Theoretical And Meta-Analytic Review. $A S A C, 115-127$.

Boyd, C. (2002). Customer violence and employee health and safety. Work, Employment and Society, 16, 151-169.

Brancu, M., Mann-Wrobel, M-, Beckham, J., Wagner, R., Elliott, A., Robbins, A., Wong, M., Berchuck, A. \& Runnals, J. (2016). Subthreshold Posttraumatic Stress Disorder: A MetaAnalytic Review of DSM-IV Prevalence and a Proposed DSM-5 Approach to Measurement. Psychological Trauma: Theory, Research, Practice, and Policy, 8(2), 222-232.

Bryman, A. \& Cramer, D. (2003). Análise de dados em ciências sociais, introdução às técnicas utilizando o SPSS para Windows. Oeiras: Celta Editora.

Caiuby, A., Lacerda, S., Quintana, M., Torii, T., \& Andreoli, S. (2012). Adaptação transcultural da versão brasileira da Escala do Impacto do Evento - Revisada (IES-R). Cadernos de Saúde Pública, 28(3), 597-603. 
Cocks, R. (1989). Trauma in the tube: the problem of railway suicide and its consequences. Stress Medicine, 5, 93-97.

Cohen, S., \& Hoberman, H. (1983). Positive events and social supports as buffers of life change stress. Journal of Applied Social Psychology, 13, 99-125.

Cothereau, C., Beaurepaire, C., Payan, C., Cambau, J.P., Rouillon, F. \& Cpnso, F. (2004). Professional and medical outcomes for French train drivers after "person under train" accidents: three year follow up study. Occupational Environmental Medicine, 61, 488-494.

Cox, T., Griffiths, A. \& Houdmont, J. (2003). Rail safety in Britain: an occupational health psychology perspective. Work \& Stress, 17(2), 103-108.

Dormann, C. \& Zapf, D. (2004). Customer-Related Social Stressors and Burnout. Journal of Occupational Health Psychology, 9(1), 61-82.

Doroga, C. \& Baban, A. (2013). Traumatic exposure and posttraumatic symptoms for train drivers involved in railway incidents. Clujul Medical, 86(2), 144-149.

Dursun, S. \& Aytac, S. (2014). The Effect of Customer Aggression on Burnout. Academic Journal of Interdisciplinary Studies, 3(4), 369-372.

EU-OSHA, EUROPEAN AGENCY FOR SAFETY AND HEALTH AT WORK (2005). Criar uma cultura de segurança e saúde no trabalho na Uniäo Europeia, síntese do relatório anual 2005. Bilbao: EU-OSHA.

EU-OSHA, EUROPEAN AGENCY FOR SAFETY AND HEALTH AT WORK (2006). Previsões de peritos sobre os riscos físicos emergentes associados à segurança e saúde no trabalho - FACTS no 60PT. Bilbao: EU-OSHA.

EU-OSHA, EUROPEAN AGENCY FOR SAFETY AND HEALTH AT WORK (2010). European Risk Observatory Report - European Survey of Enterprises on New and Emerging Risks: Managing safety and health at work. Bilbao: EU-OSHA.

EU-OSHA, EUROPEAN AGENCY FOR SAFETY AND HEALTH AT WORK (2013a). Managing stress and psychosocial risks at work. Luxembourg: Publications Office of the European Union.

EU-OSHA, EUROPEAN AGENCY FOR SAFETY AND HEALTH AT WORK (2013b). Estimar o custo dos acidentes e problemas de saúde relacionados com o trabalho - resumo executivo. Bilbao: EU-OSHA

EU-OSHA, EUROPEAN AGENCY FOR SAFETY AND HEALTH AT WORK (2014a). Cálculo do custo do stress e dos riscos psicossociais relacionados com o trabalho. Bilbao: EU-OSHA.

EU-OSHA, EUROPEAN AGENCY FOR SAFETY AND HEALTH AT WORK (2014b). Priorities for occupational safety and health research in Europe for the years 2013-2020 Summary report - (updated Jan 2014). Luxembourg: Publications Office of the European Union.

EU-OSHA, EUROPEAN AGENCY FOR SAFETY AND HEALTH AT WORK (2016a). Healthy workplaces for all ages: promoting a sustainable working life - campaign guide. Luxembourg: Publications Office of the European Union.

EU-OSHA, EUROPEAN AGENCY FOR SAFETY AND HEALTH AT WORK (2016b). Safer and healthier work at any age: Analysis report of workplace good practices and support needs of enterprises - executive summary. Bilbao: EU-OSHA.

Field, A. (2009). Descobrindo a estatística usando o SPSS. Porto Alegre: Artmed.

Grandey, A.A., Dickter, D.N.A \& Sin, H.P. (2004). The Customer is not Always Right: Customer Aggression and Emotion Regulation of Service Employees. Journal of Organizational Behavior, $25,1-22$. 
Green, J.D., Bovin, M.J., Erb, S.E., Lachowicz, M., Gorman, K.R., Rosen, R.C., Keane, T. \& Marx, B.P. (2015). The Effect of Enemy Combat Tactics on PTSD Prevalence Rates: A Comparison of Operation Iraqi Freedom Deployment Phases in a Sample of Male and Female Veterans. Psychological Trauma: Theory, Research, Practice, And Policy, 8(5), 634-640.

Horowitz, M.J., Wilner, M., \& Alverez, W. (1979). Impact of Events Scale: A measure of subjective stress. Psychosomatic Medicine, 41(3), 209-218.

IMT, INSTITUTO DA MOBILIDADE E DOS TRANSPORTES (2015). Transporte ferroviário: relatório anual de segurança - 2014. Lisboa: Instituto da Mobilidade e dos Transportes.

Johannesson, K.B., Stefanini, S., Lundin, T. \& Anchisi, R. (2006). Impact of bereavement among relatives in Italy and Sweden after the Linate airplane disaster. International Journal of Disaster Medicine, 4(3), 110-117.

Kilpatrick, D.G., Resnick, H.S., Milanak, M.E., Miller, M.W., Keyes, K.M. \& Friedman, M.J. (2013). National Estimates of Exposure to Traumatic Events and PTSD Prevalence Using DSM-IV and DSM-5 Criteria. Journal of Traumatic Stress, 26(5), 537-547.

Kumar, P., Gupta, S., Agarwal, M. \& Singh, U. (2016). Categorization and standardization of accidental risk-criticality levels of human error to develop risk and safety management policy. Safety Science, 85, 88-98.

Lemos, I.C. (2013). Acidente com comboios: Implicaçôes no desenvolvimento da perturbaçâa de stress pós-traumático nos maquinistas. Dissertação de Mestrado em Psicologia Clínica. Lisboa: ISPA.

Lunt, J. \& Hartley R. (2004). Literature Review of Post-Traumatic Stress Disorder Amongst Rail Workers. Sheffield: Work Psychology Group, Health and Safety Laboratory.

Maia, A.C. \& Fernandes, E. (2003). Epidemiologia da perturbação pós-stress traumático (PTSD) e avaliação da resposta ao trauma. In M.G. Pereira \& J. Monteiro-Ferreira (Eds.). Stress traumático: aspectos teóricos e intervenção (pp.35-54). Lisboa: Climepsi.

Maroco, J. (2010). Análise estatística com o PASW Statistics. Pêro Pinheiro: Report Number.

Maslach, C. \& Leiter, M. (2016). Understanding the burnout experience: recent research and its implications for psychiatry. World Psychiatry 15(2), 103-11.

Mehnert, A., Nanninga, I, Fauth, M. \& Schafer, I. (2012). Course and predictors of posttraumatic stress among male train drivers after the experience of 'person under the train' incidents. Journal of Psychosomatic Research, 73, 191-196.

Moura, R., Beer, M., Patelli, E., Lewis, J. \& Knoll, F. (2015). Human error analysis: Review of past accidents and implications for improving robustness of system design. In T. Nowakowski, M. Młynczak, A. Jodejko-Pietruczuk \& S. Werbińska-Wojciechowska (Eds). Safety and Reliability: Methodology and Applications (pp.1037-1046). London: Taylor \& Francis.

North, C., Pollio, D., Hong, B., Pandya, A., Smith, R. \& Pfefferbaum, B. (2015). The post disaster prevalence of major depression relative to PTSD in survivors of the 9/11 attacks on the world trade center selected from affected workplaces. Comprehensive Psychiatry, 60, 119-125.

Pereira, M.G. \& Monteiro-Ferreira, J. (2003). Stress traumático: aspectos teóricos e intervençāo. Lisboa: Climepsi.

Queirós, C., Fonseca, S., Guimarães, F. \& Martins, V. (2016). Relatório Técnico: Stress, motivação no trabalho e desgaste em profissionais que trabalham no sector ferroviário. Porto: LabRP da FPCEUP/ESTSPIPP.

Rabjohn, A. (2012). The human cost of being a 'first responder'. Journal of Business Continuity \& Emergency Planning, 6(3), 268-271. 
Ranney, M.L., Patena, J.V., Nugent, N., Spirito, A., Boyer, E., Zatzick, D. \& Cunningham, R. (2016). PTSD, cyberbullying and peer violence: prevalence and correlates among adolescent emergency department patients. General Hospital Psychiatry, 39, 32-38.

Richards, D. A. \& Schat, A.C. (2007). Attachment and Customer Aggression: An Affective Events Theory Model. ASAC, 253-269.

Saberi, R., Abbasian, H., Kashani, M.M., \& Naseri, A. (2013). Post-Traumatic Stress Disorder: A Neglected Health Concern among Commercial Motor Vehicle Drivers. International Journal of Occupational and Environmental Medicine 4(4), 185-194.

Sachser, C. \& Goldbeck, L. (2016). Consequences of the Diagnostic Criteria Proposed for the ICD-11 on the Prevalence of PTSD in Children and Adolescents. Journal of Traumatic Stress, 29(2), 120-123.

Sage, C., Brooks, S., Jones, N. \& Greenberg, N. (2016). Attitudes towards mental health and help-seeking in railway workers. Occupational Medicine, 66(2), 118-121.

Vatshelle, A. \& Moen, B. (1997). Serious on-the-track accidents experienced by train drivers: psychological reactions and long-term health effects. Journal of Psychosomatic Research, 42(1), 43-52.

Vaz Serra, A (2003). O distúrbio de stress pós-traumático. Coimbra: Gráfica de Coimbra.

Weiss, D.S. (2007). The Impact of Event Scale: Revised. In J.P. Wilson \& C.S. Tang (Eds.), Crosscultural assessment of psychological trauma and PTSD (pp. 219-238). New York: Springer.

Williams, C., Miller, J., Watson, G. \& Hunt, N. (1994). A strategy for trauma debriefing after railway suicides. Social Science \& Medicine, 38(3), 483-487.

Wilson, L.C. 2015. A systematic review of probable posttraumatic stress disorder in first responders following man-made mass violence. Psychiatry Research, 229(1-2), 21-26.

Yagil, D. (2008). When the customer is wrong: A review of research on aggression and sexual harassment in service encounters. Aggression and Violent Behavior, 13, 141-152.

Yeow, J., Ng, P., Tan, K., Chin, T. \& Lim, W. (2014). Effects of Stress, Repetition, Fatigue and Work Environment on Human Error in Manufacturing Industries. Journal of Applied Science, 14(24), 3464-3471. 